\title{
On a Gaussian Approximation to the Capacity of Wireless MIMO Systems
}

\author{
Peter J Smith* and Mansoor Shafi ${ }^{\dagger}$ \\ * Department of Electrical and Electronic Engineering \\ University of Canterbury, Private Bag 4800 \\ Christchurch, New Zealand \\ $\dagger$ Telecom New Zealand Limited, P O Box 293 \\ Wellington, New Zealand
}

\begin{abstract}
In this paper we consider the capacity of a single user $t \rightarrow r$ MIMO wireless system in a Rayleigh or Ricean fading environment. It is known that a certain central limit theorem exists which states (under certain conditions) that the distribution of the standardized capacity is asymptotically Gaussian as $r \rightarrow \infty, t \rightarrow \infty$ and $r / t \rightarrow y$ for some constant $y$. However we demonstrate the surprising accuracy of a Gaussian approximation to the capacity for virtually all values of $r$ and $t$. In order to investigate the accuracy of the Gaussian fit we derive the variance of the capacity in the Rayleigh fading case.
\end{abstract}

\section{INTRODUCTION}

Multiple Input Multiple Output (MIMO) systems have recently been a subject of intense research activity [1-3] since the fundamental information-theoretic work of Foschini [1] and Telatar [2]. In this paper we consider the capacity of a single user $t \rightarrow r$ MIMO wireless system in a Rayleigh or Ricean fading environment. Throughout we will adhere to the notation, $r$ $=$ no. of receive branches, $t=$ no. of sources, $m=\min (t, r)$ and $n=\max (t, r)$. In paricular we are interested in the capacity distribution since several authors $[1,3]$ use simulated capacity outage curves in their analyses. The exact distribution of the capacity in a Rayleigh fading environment can be found in principle (ie., the exact characteristic function is derived in [4]). However, the results are probably too complex to be of practical use. Now it is known that a certain central limit theorem exists [5] which states that the distribution of the standardized capacity is asymptotically Gaussian as $r \rightarrow \infty, t \rightarrow \infty$ and $r / t \rightarrow y$ for some constant $y$. The standardized capacity is simply the capacity shifted and scaled to have zero mean and unit variance. In other words if $C$ is the capacity variable with mean $\mu$ and standard deviation $\sigma$ then the standardized capacity is $(C-\mu) / \sigma$. The conditions required for this convergence are discussed below but assuming iid fading on all $r \times t$ paths we can use this result for both Rayleigh and Ricean fading channels. However to compute the Gaussian approximation we require the mean and variance of the capacity. For the Rayleigh case Telatar [2] has derived an exact expression for the mean and Rapajic and Popescu [6] computed the limiting value of the mean in the above sense. Neither Telatar, nor Rapajic evaluate the variance of channel capacity and so this is done in the Appendix. For the Ricean case no results are available and we simply obtain simulated estimates of the mean and variance to scope out the accuracy of the Gaussian approximation.

In this paper we show that:
1) the channel capacity in both types of fading can be accurately modelled by a Gaussian distribution. For the Rayleigh fading case the exact mean and variance of the capacity are given for any numbers of transmit and receive antennas.

2) The variance of the channel capacity is not sensitive to the number of antennas and is mainly influenced by the $S N R$. A closed form formula for the variance is developed in the Appendix.

In summary, the Gaussian approximation to channel capacity is a simple and powerful tool to enable engineering estimates of system capacity, total throughput and capacity outage probability. The rest of the paper is laid out as below. In Section II we review the relevant results in the literature. In Section III we discuss central limit theorems for the capacity and provide the methodology for the Gaussian approximation. In Section IV results are given and in Section $\mathrm{V}$ some conclusions are presented.

\section{BACKGROUND}

Consider a transmission system where each user transmits simultaneously via $t$ antennas and reception is via $r$ antennas. The total power of the complex transmitted signal $\mathbf{s}(t)$ is constrained to $P$ regardless of the number of antennas. The received signal $\mathbf{r}(t)$ is given by:

$$
\mathbf{r}(t)=\mathbf{H} \mathbf{s}(t)+\mathbf{n}(t)
$$

where $\mathbf{H}$ is a $(r \times t)$ complex channel gain matrix. For Rayleigh fading, the entries in $\mathbf{H}$ are $i . i . d$, complex, zero mean Gaussians with unit magnitude variance. For Ricean fading the entries are $i . i . d$, complex Gaussians with a non-zero real mean of $\mu$ and the real and imaginary components have variance $\sigma^{2}$. To ensure the total power condition is satisfied we have $\mu^{2}+2 \sigma^{2}=1$. In (1), $\mathbf{n}(t)$ is a complex $r$ dimensional AWGN vector, with statistically independent components of identical power $\sigma_{n}^{2}$ at each of the $r$ receive branches. We assume $\sigma_{n}^{2}=1$ without loss of generality. The relevant capacity for such a channel is expressed as

$$
C=\log _{2}\left(\operatorname{det}\left[\mathbf{I}_{r}+(P / t) \mathbf{H H}^{\dagger}\right]\right) \quad b p s / H z
$$

where $\dagger$ denotes transpose conjugate and $\mathbf{I}_{n}$ denotes an $n \times n$ identity matrix. 
The calculation of the mean capacity involves rewriting the expectation in terms of the eigenvalues $\lambda_{1} \ldots \lambda_{m}$ of $\mathbf{W}$ where

$$
\mathbf{W}= \begin{cases}\mathbf{H H}^{\dagger} & r<t \\ \mathbf{H}^{\dagger} \mathbf{H} & r \geq t\end{cases}
$$

With this approach an exact calculation of the mean capacity [2] yields :

$$
\langle C\rangle=\int_{0}^{\infty} \log (1+P \lambda / t) \sum_{k=0}^{m-1} \frac{k ! \lambda^{n-m} e^{-\lambda}}{(k+n-m) !}\left[L_{k}^{n-m}(\lambda)\right]^{2} d \lambda
$$

where $L_{k}^{n-m}(x)$ are generalised Laguerre polynomials of order $k$.

Note that Rapajic [6] derives a closed form expression for the limiting value of the mean capacity as $r, t \rightarrow \infty$ and $\mathrm{r} / \mathrm{t}$ is held constant. This work, as well as Foschini's [1] and Telatar's [2], shows that the mean capacity is extremely well approximated by a linear function of $m$. Since the mean grows with $m$ and the variance stabilizes, see below, the coefficient of variation of the capacity becomes extremely small as $m$ grows.

\section{Methodology}

In [5] Girko gives a central limit theorem for the capacity in (2) which requires that the elements of $\mathbf{H}$ are iid zero-mean with finite moments up to order $4+\delta$ for some $\delta>0$. This is equally valid for both Rayleigh and Ricean fading and so we simply require $E(C)$ and $\operatorname{Var}(C)$ to fit the Gaussian approximation. For the Rayleigh case the mean was given exactly by Telatar [2], see (8), and the limiting value by Rapajic and Popescu [6]. The exact variance is derived in the Appendix following Telatar's approach and is given below:

$$
\begin{aligned}
\operatorname{Var}(C) & =m \int_{0}^{\infty} w^{2}(\lambda) p(\lambda) d \lambda- \\
& \sum_{i=1}^{m} \sum_{j=1}^{m}\left[\frac{(i-1) !(j-1) !}{(i-1+n-m) !(j-1+n-m) !}\right] \times \\
& \left\{\int_{0}^{\infty} \lambda^{n-m} e^{-\lambda} L_{i-1}^{(n-m)}(\lambda) L_{j-1}^{(n-m)}(\lambda) w(\lambda) d \lambda\right\}^{2}
\end{aligned}
$$

where $w(\lambda)=\log (1+P \lambda / t), L_{i}^{(r)}(x)$ is a generalised Laguerre polynomial and

$$
p(\lambda)=m^{-1} \sum_{i=1}^{m} \frac{(i-1) !}{(i-1+n-m) !} \lambda^{n-m} e^{-\lambda} L_{i-1}^{(n-m)}(\lambda)^{2}
$$

Hence the variance can be found by several single numerical integrations via (5). Due to the complexity of (5) it would be desirable to approximate the variance, perhaps by a limiting approach such as in [2,6]. To date, no such result is available although the case where $\mathbf{H}$ is a real Gaussian matrix has been derived in [7]. For the Ricean case no results are available on the mean or variance and we simply obtain simulated estimates to scope out the accuracy of a Gaussian approximation.

\section{Results}

Figures 1-3 are for the case of Rayleigh fading and Figure 4 considers the Ricean case. Figure 1 shows the behaviour of the capacity variance as the number of antennas and SNR vary for the $r=t$ case. It appears that the variance stabilises as $m$ increases for any SNR value although this stabilisation occurs more rapidly for small SNR. These experimental results suggest that a limiting variance exists as $m \rightarrow \infty$. This is proven for the case of real Gaussian $\mathbf{H}$ matrices in [7].

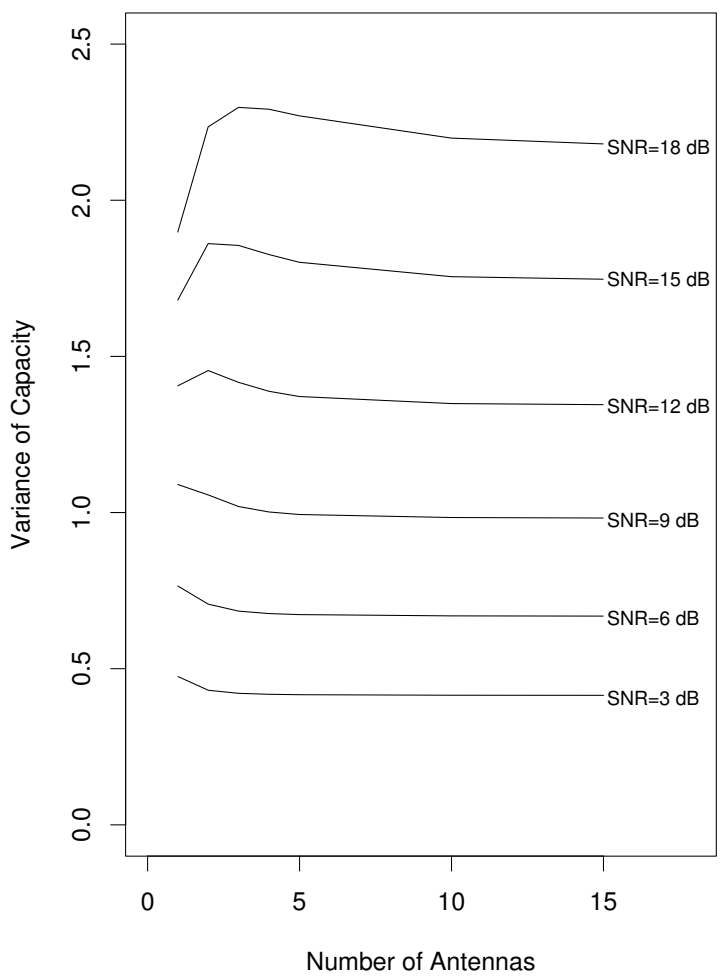

Fig. 1. Variance of capacity vs antenna number for $\mathrm{r}=\mathrm{t}$

Gaussian approximations to the capacity distribution are now investigated using analytic results for the mean and variance. Figures 2-3 show the accuracy of a Gaussian approximation to the reliability function or $\operatorname{ccdf}\left(P(C)>c_{0}\right)$. The Gaussian approximation does remarkably well over the whole range of $r$ and $t$ values considering the CLT only offers convergence in the limit. When $m \geq 5$ the Gaussian approximation is virtually indistinguishable from the simulated curve. However 
even the worst fits, $r=t=1$ in Figure 2 and $(r=2, t=4)$ in Figure 3 are quite respectable. When the SNR is reduced the fits are even better.

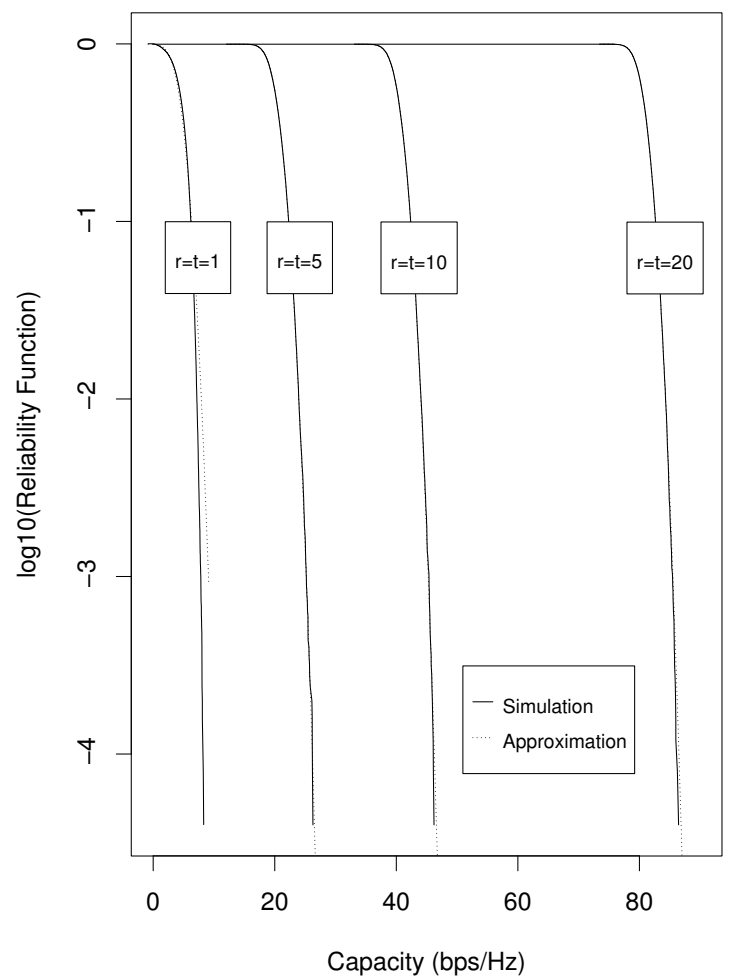

Fig. 2. Comparison of simulated capacity with normal approximation for $r=t$ $(\mathrm{SNR}=15 \mathrm{~dB})$

In Figure 4 we plot the reliability function for Ricean fading and the Gaussian approximation. The parameters used are $P=$ $15 d B$ and $\mu^{2}=2 \sigma^{2}=1 / 2$. As for the Rayleigh case the fit is remarkably good over a whole range of antenna numbers.

\section{CONCLUSiOnS}

We have derived the variance of the capacity of a MIMO system in Rayleigh fading and hence have allowed an investigation of the accuracy of a Gaussian approximation to capacity foreshadowed by various central limit theorems. It is interesting that the capacity variance appears to converge to a limit independent of absolute antenna numbers but dependent on the ratio $r / t$. The Gaussian approximation itself is surprisingly good, even in the worst cases (high SNR, low $m$ ) giving satisfactory results. For Ricean fading also the Gaussian approximation is very good although impractical at this stage since the mean and variance are unknown.

\section{APPENDIX}

In this Appendix a brief derivation of the variance of the capacity is given. We follow the derivation of the mean capacity given by Telatar [2] and extend this approach to the vari-

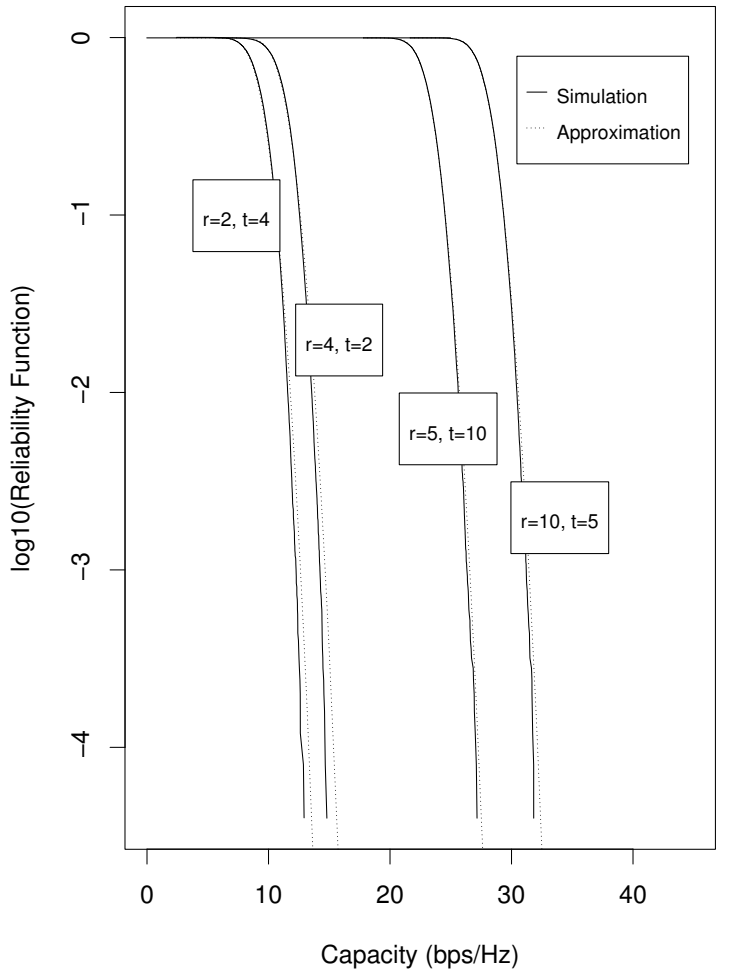

Fig. 3. Comparison of simulated capacity with normal approximation for $\mathrm{n}=2 \mathrm{~m}(\mathrm{SNR}=15 \mathrm{~dB})$

ance. Let $\lambda_{1}, \lambda_{2}, \ldots, \lambda_{m}$ denote the eigenvalues of $\mathbf{W}$ from (3). Then from (2) we have

$$
C=\sum_{i=1}^{m}\left(1+\frac{P \lambda_{i}}{t}\right)
$$

The variance of $C$ is given by

$$
\begin{aligned}
& \operatorname{Var}(C)=m \operatorname{Var}(\log (1+P \lambda / t))+ \\
& m(m-1) \operatorname{Cov}\left(\log \left(1+P \lambda_{1} / t\right), \log \left(1+P \lambda_{2} / t\right)\right)
\end{aligned}
$$

where $\lambda$ is a randomly selected eigenvalue and $\left(\lambda_{1}, \lambda_{2}\right)$ is a pair of randomly selected (distinct) eigenvalues. Using the notation $w(\lambda)=\log (1+P \lambda / t)$ we have

$$
\begin{aligned}
\operatorname{Var}(C) & =m\left[E\left(\omega^{2}(\lambda)\right)-E(\omega(\lambda))^{2}\right]+ \\
& m(m-1)\left[E\left(\omega\left(\lambda_{1}\right) \omega\left(\lambda_{2}\right)\right)-E(\omega(\lambda))^{2}\right]
\end{aligned}
$$

The main difficulty in (8) is the evaluation of $E\left(\omega\left(\lambda_{1}\right) \omega\left(\lambda_{2}\right)\right)$ for which we need the joint density of $\lambda_{1}, \lambda_{2}$. Telatar [2] gives the joint density of $\lambda_{1} \lambda_{2} \ldots, \lambda_{m}$ as 


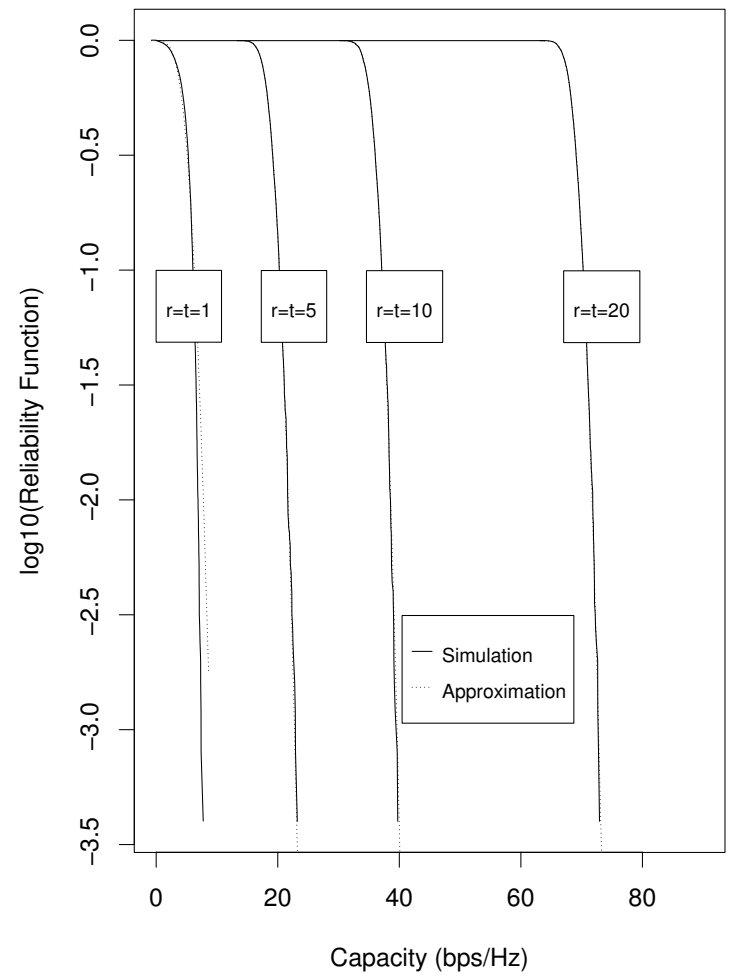

Fig. 4. Comparison of simulated capacity with normal approximation for Ricean case $(\mathrm{SNR}=15 \mathrm{~dB})$

$$
\begin{array}{r}
p_{\lambda}\left(\lambda_{1} \lambda_{2} \ldots, \lambda_{m}\right)=[m !]^{-1} \sum_{\alpha, \beta}(-1)^{\operatorname{per}(\alpha)+\operatorname{per}(\beta)} \times \\
\Pi_{i=1}^{m} \phi_{\alpha_{i}}\left(\lambda_{i}\right) \phi_{\beta i}\left(\lambda_{i}\right) \lambda_{i}^{n-m} e^{-\lambda_{i}}
\end{array}
$$

where the sum is over all possible permutations $\alpha, \beta$ of $\{1,2, \ldots, m\}, \operatorname{per}(\cdot)$ denotes the sign of the permutation and $\phi_{k}(\lambda)$ is given by

$$
\phi_{k}(\lambda)=\left\{\frac{(k-1) !}{(k-1+n-m) !}\right\}^{\frac{1}{2}} L_{k-1}^{(n-m)}(\lambda)
$$

where $L_{k-1}^{(n-m)}(\lambda)$ is a generalised Laguerre polynomial. Since $\lambda_{1}, \lambda_{2}, \ldots, \lambda_{m}$ are unordered we can obtain the joint density of $\lambda_{1}, \lambda_{2}$ by integrating (9) over $\lambda_{3}, \lambda_{4}, \ldots, \lambda_{m}$ and using the orthogonality relationship of Laguerre polynomials. This approach gives the joint density of $\lambda_{1}, \lambda_{2}$ as

$$
\begin{aligned}
p\left(\lambda_{1}, \lambda_{2}\right) & =[m(m-1)]^{-1} \sum_{i=1}^{m} \sum_{j \neq i}\left(\lambda_{1} \lambda_{2}\right)^{n-m} e^{-\left(\lambda_{1}+\lambda_{2}\right)} \\
& \times \frac{(i-1) !(j-1) !}{(i-1+n-m) !(j-1+n-m) !} \\
& \times\left\{L_{i-1}^{(n-m)}\left(\lambda_{1}\right)^{2} L_{j-1}^{(n-m)}\left(\lambda_{2}\right)^{2}-\right. \\
& \left.L_{i-1}^{(n-m)}\left(\lambda_{1}\right) L_{j-1}^{(n-m)}\left(\lambda_{1}\right) L_{i-1}^{(n-m)}\left(\lambda_{2}\right) L_{j-1}^{(n-m)}\left(\lambda_{2}\right)\right\}
\end{aligned}
$$

With a little rearrangement (11) can be rewritten as

$$
p\left(\lambda_{1}, \lambda_{2}\right)=\frac{m}{m-1} p\left(\lambda_{1}\right) p\left(\lambda_{2}\right)-\frac{1}{m(m-1)} K\left(\lambda_{1}, \lambda_{2}\right)^{2}
$$

where $p(\lambda)$ is the density of an arbitrary eigenvalue given by Telatar [2] as

$$
p(\lambda)=m^{-1} \sum_{i=1}^{m} \frac{(i-1) !}{(i-1+n-m) !} \lambda^{n-m} e^{-\lambda} L_{i-1}^{(n-m)}(\lambda)^{2}
$$

and

$$
\begin{aligned}
K\left(\lambda_{1}, \lambda_{2}\right) & =\sum_{i=1}^{m} \frac{(i-1) !}{(i-1+n-m) !}\left(\lambda_{1} \lambda_{2}\right)^{(n-m) / 2} \\
& \times e^{-\left(\lambda_{1}+\lambda_{2}\right) / 2} L_{i-1}^{(n-m)}\left(\lambda_{1}\right) L_{i-1}^{(n-m)}\left(\lambda_{2}\right)
\end{aligned}
$$

Now we can turn to the calculation of $E\left(\omega\left(\lambda_{1}\right) \omega\left(\lambda_{2}\right)\right)$ since $m(m-1) E\left(\omega\left(\lambda_{1}\right) \omega\left(\lambda_{2}\right)\right)$ is given by

$$
m^{2} E(\omega(\lambda))^{2}-\int_{0}^{\infty} \int_{0}^{\infty} K\left(\lambda_{1}, \lambda_{2}\right)^{2} \omega\left(\lambda_{1}\right) \omega\left(\lambda_{2}\right) d \lambda_{1} d \lambda_{2}
$$

Substituting (13) in (8) gives

$$
\begin{aligned}
\operatorname{Var}(C) & =m \int_{0}^{\infty} \omega^{2}(\lambda) p(\lambda) d \lambda \\
& \times \int_{0}^{\infty} \int_{0}^{\infty} K\left(\lambda_{1}, \lambda_{2}\right)^{2} \omega\left(\lambda_{1}\right) \omega\left(\lambda_{2}\right) d \lambda_{1} d \lambda_{2}
\end{aligned}
$$

The integrals in (14) appear to be intractable in closed form. The double integral can also be evaluated numerically or we can take the summations in $K\left(\lambda_{1}, \lambda_{2}\right)^{2}$ outside the integrals to give 


\section{REFERENCES}

$$
\begin{aligned}
\operatorname{Var}(C) & =m \int_{0}^{\infty} \omega^{2}(\lambda) p(\lambda) d \lambda- \\
& \sum_{i=1}^{m} \sum_{j=1}^{m}\left[\frac{(i-1) !(j-1) !}{(i-1+n-m) !(j-1+n-m) !}\right] \\
& \times\left\{\int_{0}^{\infty} \lambda^{n-m} e^{-\lambda} L_{i-1}^{(n-m)}(\lambda) L_{j-1}^{(n-m)}(\lambda) \omega(\lambda) d \lambda\right\}^{2}
\end{aligned}
$$

[1] G.J. Foschini and M.J. Gans, "On limits of wireless communication in a fading environment when using multiple antennas," Wireless Personal Communications, Vol.6, No.3, p.311-335, March 1998.

[2] E. Telatar, Capacity of multi-antenna Gaussian channels, Technical Report \# BL0112170-950615-07TM, AT \& T Bell Laboratories, 1995.

[3] D-S. Shiu, Wireless Communication using Dual Antenna Arrays, Kluwer Academic Publishers, Boston, 1999.

[4] P.J. Smith, S. Roy and M. Shafi, "On the capacity of MIMO systems with correlated fading and/or space time processing," unpublished.

[5] V.L. Girko, "A refinement of the central limit theorem for random determinants," Theory of Probability and its Applications, Vol.42, No.1, p.121-129, 1997.

[6] P.B. Rapajic and D. Popesu, "Information capacity of a random signature multiple-input multiple-output channel," IEEE Transactions on Communications, Vol.48, No.8, p.1245-1248, August 2000.

[7] V.L. Girko and P.J. Smith, "The variance of the logarithm of the regularized random Gram matrix and its application to the capacity of MIMO systems," unpublished. 